CAPSULE COMMENTARIES

\title{
Capsule Commentary on Hoffman et al., Brief Training of Student Clinicians in Shared Decision Making: A Single-Blind Randomized Control Trial
}

\author{
Madeline Sterling, MD, MPH \\ Resident of Internal Medicine/Primary Care, New York Presbyterian - Weill Cornell Medical College, New York, NY, USA.
}

J Gen Intern Med 29(6):891

DOI: $10.1007 / \mathrm{s} 11606-014-2800-6$

(c) Society of General Internal Medicine 2014

$\mathrm{T}$ he Institute of Medicine highlighted shared decisionmaking (SDM) over a decade ago. ${ }^{1}$ Since then, more than 80 randomized trials have been published, many demonstrating that involving the patient in decisions and improving provider communication are associated with positive healthcare quality markers and overall morbidity and mortality outcomes. ${ }^{2}$ Few have involved multi-disciplinary practitioners; the trial by Hoffman et al. ${ }^{3}$ evaluated the effectiveness of a brief intervention on facilitating shared decision making (SDM) among student clinicians (of medicine, physiotherapy and occupational therapy) from two Australian universities.

Based on valid, objective measures of video recorded interactions with simulated patient encounters, they found that their intervention improved performance of student healthcare providers in several important outcomes, including SDM communication skills, self-confidence in SDM skills, SDM attitudes and involving patients in decision making. An important limitation of this study is that it is based on simulated patients rather than live patients and the impact of the intervention was measured shortly after the intervention. However, its rigorous methods and robust evaluations suggest that modest interventions can yield significant improvement in learner SDM skills.

A very important finding is that participants had high confidence at baseline in their SDM skills; a confidence that was not borne when objectively measured. This has been seen in other studies, whereby learners express overconfidence in their own communication skills-which begs the question: do residents and physicians do the same?

One of the core competencies for US residencies is interpersonal and communication skills. This study high- lights tools that are available for measuring learner competencies in shared decision making (OPTION scale ${ }^{4}$ ) and in eliciting patient preferences $\left(\mathrm{ACEPP}^{5}\right)$. The authors are willing to share their training materials for programs that want to adopt formal curricula in shared decision making. There are a number of potential research questions that could be asked, such as the impact of health literacy (and numeracy), language/culture concordance between the provider and the patient and whether or not training in SDM persists over time, and whether knowledge gained from artificial simulated encounters transfers to inpatient and outpatient settings.

Conflict of Interest: The author declares that she does not have a conflict of interest.

Corresponding Author: Madeline Sterling, MD, MPH; Resident of Internal Medicine/Primary CareNew York Presbyterian Weill Cornell Medical College, New York, NY, USA (e-mail: mrs9012@nyp.org).

\section{REFERENCES}

1. National Research Council. Crossing the quality chasm; a new health system for the 21 st century. Washington, DC: National Academies Press; 2001.

2. Barry MJ, Edgman-Levitan, S. Shared decision making - the pinnacle of patient-centered care. N Engl J Med. 2012;366:780-781.

3. Hoffmann TC, Bennett S, Tomsett C, Del Mar C. Brief training of student clinicians in shared decision making: as single-blind randomized controlled trial. J Gen Intern Med. 2014; doi:10.1007/s11606-014-2765-5.

4. Elwyn G, Edwards A, Wensing M, Hood K, Atwell C, Grol R. Shared decision making: developing the OPTION scale for measuring patient involvement. Qual Saf Health Care. 2003;12(2):93-9.

5. Shepherd HL, Barratt A, Trevena LJ, McGeechan K, Carey K, Epstein RM, Butow PN, Del Mar CB, Entwistle V, Tattersall MH. Three questions that patients can ask to improve the quality of information physicians give about treatment options: a cross-over trial. Patient Educ Couns. 2011;84(3):379-85. 